OUTP -96 - 66-P

DSF $53 / 96$

hep-th/9611040

November 1996

\title{
Quantum Phase Space From String Solitons
}

\author{
Fedele LizzI" and Nick E. Mavromatos] \\ University of Oxford, Theoretical Physics, \\ 1 Keble Road, Oxford, OX1 3NP, UK.
}

\begin{abstract}
In this paper we view the $\sigma$-model couplings of appropriate vertex operators describing the interaction of string matter with a certain type of string solitons (0-branes) as the quantum phase space of a point particle. The $\sigma$-model is slightly non critical, and therefore one should dress it with a Liouville mode. Quantization is achieved by summing over worldsheet genera (in the pinched approximation). To leading order in the coupling constant expansion, the quantization reproduces the usual quantum mechanical commutator. We attempt to go beyond leading order, and we reproduce the generalized string uncertainty principle.
\end{abstract}

\footnotetext{
1 On leave from Università di Napoli Federico II, Dept. of Physical Sciences, Pad. 19 Mostra d'Oltremare, 80125 Naples, Italy. e-mail: lizzi@na.infn.it

2 P.P.A.R.C. Advanced Fellow. e-mail: mavroman@thphys.ox.ac.uk
} 


\section{Introduction.}

The concepts of space and time and its quantization are still far from being understood. One of the most promising hopes for a reconciliation of quantum Gravity with quantum mechanics seems, at present, available within the framework of string theory. The underlying two-dimensional conformal field theory structure of strings provide a field-theoretic framework for a consistent formulation of target-space quantum gravity as an effective theory of strings below the Planck (or String) scale.

One of the most fruitful ways to look at string theories, in turn, is to see them as two dimensional $\sigma$-models, that is field theories on a two dimensional 'space-time', the world-sheet, on which some fields are defined, those fields are the coordinates of a 'target' space time on which the string moves.

Admittedly, this world-sheet description of string theories may not be sufficient for a complete non-perturbative description of quantum space time effects. Target-space instantons in certain string backgrounds may not have their analogues as world-sheet elevated objects of a conformal field theory [四]. However, for most purposes a world-sheet conformal field theory may prove sufficient.

In this paper we would like to go a step further. We will consider the target space of the fields defined on the world sheet as the scaffolding necessary to build solitons. We will then consider as coordinates of our phase space the $\sigma$-model couplings of the vertex operators of these solitons. This idea is reminiscent of the world-sheets for world-sheets idea of M.B. Green [2], in which the target space of one (two-target-space-dimensional) string theory is considered as the world-sheet of the 'next' theory. Here it is rather (critical-dimension) solitonic stringy space times for phase-spaces. What we will try to do, is to quantize the coordinates of this theory space. This is a space with a very rich structure, as the work of Zamolodchikov on the renormalization group flow has shown [3]. A quantization of such a space is achieved by summing over topologies on the world sheet 田, 5.

Our action will be the usual $\sigma$-model action for strings with the addition of terms describing the boundary conditions appropriate for a soliton (D-brane) background [6, 77. This action has infinities coming from the coupling of string matter to the soliton, corresponding (from the scaffolding space point of view) to the recoil of the D-brane due to scattering [8, 9, 10, 11]. To cancel these infinities one has to add these recoil operators to the action. It turns out [9, 11] that the recoil operators, being solutions of a degenerate hypergeometric equation, come in logarithmic pairs [12, 13]. It is the couplings of this pair of operators which play the role of position and momentum in the approach we propose here. The appearance of a phase space is due to the mixing of these logarithmic operators. 
Quantization is then achieved by observing that, due to renormalization, the theory is slightly non critical [14, 15]. To restore criticality at the scaffolder level[, one adds the Liouville field. In fact, a finite scale transformation on the world-sheet, is a Galilean transformation in our phase space. Thus one could also see the zero mode of the Liouville field as the evolution parameter [15, 16, 11, 17]. The renormalization group flow for the Liouville field satisfies the so-called Helmholtz conditions, a set of conditions which, if satisfied, ensure the possibility of a canonical quantization 18, 4. We verify that the couplings of the logarithmic operators of the D-brane satisfy these conditions, and therefore are canonically quantized in theory space.

The quantization is achieved by a summation over world-sheet genera, albeit in the approximation of pinched world sheets [4, 5, 8, 9, 19]. To leading order in the $\sigma$-model coupling constant expansion, the canonical quantization reproduces the usual quantum phase space with position and momenta having a constant commutator. We have however attempted to go beyond the leading approximation, by incorporating stringy effects. We find corrections to the commutator which reproduce the generalized string uncertainty principle [20]. Let us however stress that our approach is different. There, the case was made that trivial world-sheet topologies gave the dominant contribution, due to some sort of 'string asymptotic freedom'. In our approach fluctuating topologies are crucial.

In this paper we are not aiming at giving a rigorous account of the quantization of solitons in string theory. Instead, we would like to suggest new ways of using string theories, and in particular the non-critical ones, to explore the connections between space and quantization. In this respect, we would like to mention that our use of Liouville dynamics will be physically different from that of ref. [15, 5]. There, the Liouville field was identified with the scaffolder time $X^{0}$, which is not the case in this work.

The structure of the article is as follows. In section 2 we review briefly the $\sigma$-model formalism describing interaction of D-branes with string matter, and introduce the logarithmic operators. In section 3 we discuss how the space of the coupling constants pertaining to these operators may be viewed as a quantum phase space. In section 4 we present a $\sigma$-model path-integral approach to quantization, summing over pinched worldsheet topologies. In section 5 we derive the phase space commutators and reproduce the generalized uncertainty principle. In section 6 we collect some concluding thoughts. Finally, some technical details related to logarithmic operators are collected in the appendix.

\footnotetext{
${ }^{3}$ By scaffolder point of view we mean the (usual) point of view in which the coordinates of space time are the fields $X$ defined on the two dimensional world sheet.
} 


\section{D-branes in $\sigma$-model Formalism}

Dirichlet-Branes (or D-branes) are solitonic states in open string theories which appear if one considers $10-(p+1)$ of the (critical-dimension) (super)string coordinates to satisfy Dirichlet (rather than Neumann) boundary conditions at the world-sheet boundary [6]. From the point of view of the scaffolding space time, then, there are the zero modes of some target-space (collective) coordinates which do not change with the world sheet coordinate $\tau$. There is, therefore, an object which can be interpreted as a soliton at rest.

Such structures encode enormous information about non-perturbative string symmetries, such as duality, connections among different string theories etc. In this section we review the relevant aspects of the D-brane formalism in the $\sigma$-model approach. Far from being complete in this discussion, we refer the interested reader to the very rich literature on the subject, for example [6].

In this paper we will consider only the case in which $p=0$, that is the case of the so called 0-brane. In this case, seen from the scaffolding point of view, the soliton is zero dimensional and appears as a particle at rest (with an opportune choice of reference frame).

The particular boundary conditions describing the D-brane can be given a $\sigma$-model lagrangian formulation by considering a world-sheet boundary operator (see [7] and references therein):

$$
V_{D}=\int_{\partial \Sigma} x_{i} \partial_{n} X^{i}+v_{i} X^{0} \partial_{n} X^{i}
$$

where $n$ denotes the normal derivative on the boundary of the world sheet $\partial \Sigma$, which at tree level is assumed to have the topology of a disk of size $L ; X^{i}, i=1, \ldots 9$ denote the collective excitations of the brane satisfying Dirichlet boundary conditions on the world-sheet boundary, while $X^{0}$ satisfies standard Neumann boundary conditions,

$$
X^{i}(\text { boundary })=0, i=1, \ldots, 9 \quad \partial_{n} X^{0}(\text { boundary })=0
$$

The two couplings $x_{i}$ and $v_{i}$ in (1) have the meaning of the initial position and velocity of the free soliton respectively. Here and in the following we shall limit ourselves to the case of a flat world volume. Of course the lower case $x$ 's, which are, from a two dimensional point of view, couplings should not be confused with the $X$ 's which instead are fields. 


\section{$2.1 \quad$ D-branes Coupled with String Matter}

We will now consider the interaction of the soliton with string matter, and in particular, in a perturbative spirit, with the lowest lying states of the string, tachyons in the bosonic casef.

Let us therefore consider for the moment the following action:

$$
S_{\sigma}=\int_{\Sigma}\left[\partial X^{\mu} \bar{\partial} X^{\nu} \eta_{\mu \nu}+T(X)\right]+\int_{\partial \Sigma} x_{i} \partial_{n} X^{i}+v_{i} X^{0} \partial_{n} X^{i}
$$

where $\eta_{\mu \nu}$ is a (critical-dimension) flat Minkowski space-time metric, and $T(X)$ denotes a bulk (closed string) matter operator, which for the case of bosonic strings is taken to be the tachyon.

We immediately encounter an infinity in the anulus correction to the tachyon propagator in the presence of a D-brane, which expresses quantum effects in the interaction of matter (tachyons) with the soliton (D-brane) background. One starts by considering the scattering of a closed string tachyon (matter) state off a 0 brane. For this purpose, it is sufficient to consider the anulus amplitude of two closed-string vertex operators $V(k)$, which must be integrated over the propagating open string. This computation may be performed using the operator formalism, in which one evaluates $\operatorname{Tr} V\left(k_{1}\right) \Delta V\left(k_{2}\right) \Delta$, with $\Delta^{-1} \equiv L_{0}-1$, where $L_{0}$ is the Virasoro operator.

The part of this computation that is relevant for our purposes is the one due to the world-sheet zero modes [8, 9, 10]. Writing $\Delta \equiv \int_{0}^{1} d q q^{L_{0}-2}, L_{0}=2 p^{2}+N$, where $N$ is the string level number, and picking out the $N=0$ part, we find the following contribution to the anulus amplitude:

$$
\mathcal{A}=\int d p<p\left|\exp \left(-i k_{1, i}^{0} X^{i, 0}\right) q_{1}^{-2\left(p^{0}\right)^{2}} \exp \left(-i k_{2, i}^{0} X^{i, 0}\right) q_{2}^{-2\left(p^{0}\right)^{2}}\right| p>
$$

where the integral is over momentum eigenstates of the Virasoro operator $L_{0}$ 巳, the superscript 0 denotes time components in the scaffolder $\sigma$-model, and $q$ is the modular

\footnotetext{
${ }^{4}$ As well known, bosonic strings suffer from the usual tachyonic instabilities. Supersymmetry gets rid of such states, and also stabilizes the solitonic backgrounds. Since in what follows we are interested in the bosonic sector of the theory, we will ignore the fermionic sector altogether. Inclusion of this sector would probably induce a spin structure in our phase space.

${ }^{5}$ Here one assumes diagonalizability of $L_{0}$ in the space of states, which is justified in a leadingdivergence approximation [11, 17]. In the case of D-branes there are subleading divergences coming from non-diagonalizable parts of $L_{0}$. Such non-diagonalizability is a generic feature of a pair of logarithmic operators 13, 9, 11], leading to non-trivial mixing. In the above analysis we determine one member of the pair, namely the one associated with leading divergences. Later, we determine the other member by applying generic conformal field theory analysis [11.
} 
parameter of the anulus. The trace over the zero modes yields the generic form [10]:

$$
\mathcal{A} \sim \delta\left(k_{1}^{0}+k_{2}^{0}\right) \sqrt{\frac{1}{\log \left(q_{1}\right)}} f\left(q_{2}, k_{2}^{0}\right)
$$

The $\delta\left(k_{1}^{0}+k_{2}^{0}\right)$ function arises from integrating over the zero modes of $\sigma$ model fields $X^{0}(z, \bar{z})$ in a standard fashion. The amplitude (田, 国) is pathological, in the sense that it is divergent as $q_{1} \rightarrow 0$, and requires regularization. It is this regularization which modifies the relation (5) in order to ensure momentum conservation in the presence of string loop effects. Seen from a scaffolder point of view, such effects correspond to the recoil of the soliton in the scattering process.

The pathological behaviour in the limit $q_{1} \rightarrow 0$ corresponds to the pinched-anulus configuration:

$$
\mathcal{A} \sim g_{s t} \int_{q \sim 0} \frac{d q_{1}}{q_{1} \sqrt{8 \pi \log \left(q_{1}\right)}} A_{\text {disk }}\left(k_{1}, k_{2}\right)
$$

where $A_{\text {disc }}\left(k_{1}, k_{2}\right)=<V\left(k_{1}\right) V\left(k_{2}\right) V^{i} V^{i}>$ is the tree-level disc amplitude, with $\left\{V_{i}\right\}$ denoting a complete set of string states.

One may cancel these infinities by adding to the action the so called recoil operators. These operators where introduced in [8, 9, 11, 10] to describe the back reaction (the recoil) of the soliton when two tachyons scatter against it.

In this paper we are not interested in the role of the recoil operators from the point of view of scattering in the scaffolding space-time per se, but rather in the rôles of their couplings as generating quantum operators of a phase space. However we find it useful for the convenience of the reader to review the basic properties of these operators and their origin. We do this in the next subsection and in the appendix. Further details can be found in [1, 11, 10, 17].

\subsection{D-branes and logarithmic operators}

As argued in refs. [9, 19, 11], in order to cancel the one-loop infrared divergence (6) one must add the following tree-level closed-string (bulk) operator counterterm

$$
\delta \mathcal{A}=\int d^{2} z \partial_{\alpha}\left(f\left(X^{0}\right) \partial^{\alpha} X^{i}\right)
$$

which contributes on the boundary. Its form is determined by general properties of soliton backgrounds in string theory [9], and the function $f\left(X^{0}\right)$ in (7) is determined by requiring 
that the above operator reproduce the infinities of the anulus amplitude (6). The result is [10]:

$$
\left.V_{i m p} \equiv \int d^{2} z \partial_{\alpha}\left(\left[u_{i} X^{0}\right] \Theta\left(X^{0}\right) \partial_{\alpha} X^{i}\right)=\int d \tau u_{i}\left(X^{0} \Theta\left(X^{0}\right)\right) \partial_{n} X^{i}\right) ; \quad i=1, \ldots, 9 .
$$

The step-function operator in (8) needs to be defined properly, and we adopt the integral representation [11]:

$$
\Theta_{\epsilon}\left(X^{0}\right)=-i \int_{-\infty}^{\infty} \frac{d q}{q-i \epsilon} e^{i q X^{0}} \quad, \quad \epsilon \rightarrow 0^{+}
$$

where $\epsilon$ is a regulator parameter. From the scaffolder point of view, the coupling constant $u_{i}$ may be considered as the change in the velocity of the brane due to recoil [10, 11]. This may be obtained by imposing overall conformal invariance of the anulus and disc amplitudes [8]. As we shall discuss below, the cancellation of tree and anulus divergences in our case is formally different from the approach of ref. [8]. However, the basic qualitative features remain the same, and the above cancellation requires [8, 10]

$$
u_{i}=8 \sqrt{2} \pi g_{s}\left(k_{1}+k_{2}\right)_{i}
$$

which expresses momentum conservation. This interpretation of (10) is consistent with the fact that the soliton mass is proportional to $1 / g_{s}$, confirming the interpretation of $u_{i}$ as the D-brane velocity.

But this is not the end of the story. Analysis of the operator product of the operator (8) reveals that it is a logarithmic operator [12]

$$
V_{i m p}(\tau) V_{i m p}(0) \sim \frac{\log \tau}{\tau^{2}}
$$

Logarithmic operators, however, being related to degenerate solutions of hypergeometric equations, are known to come in pairs $C$ and $D$ [12, 13]. We discuss the appearance and the properties of such logarithmic operator pairs in the appendix.

To identify the pair in our case let us concentrate, for convenience, on the $X^{0}$ dependent parts. It is clear that $X^{0} \Theta\left(X^{0}\right)$ appearing in (8) plays the role of the logarithmic $D$ operator [11]. Using the integral representation (9) one may write it in the form

$$
D_{\epsilon}=X^{0} \Theta_{\epsilon}\left(X^{0}\right)=-i \int_{-\infty}^{\infty} \frac{d q}{q-i \epsilon} X^{0} e^{i q X^{0}}=-\int_{-\infty}^{\infty} \frac{d q}{(q-i \epsilon)^{2}} e^{i q X^{0}}
$$

where we have integrated by parts. 
To find the corresponding $C$ operator we study the operator product of $D_{\epsilon}$ (12) with the stress-energy tensor of the $\sigma$-model. Using the fact that the conformal dimension of the operator $e^{i q X^{0}}$ is $q^{2} / 2$ one gets 11

$$
T(w) D_{\epsilon}(z)=-\frac{\epsilon^{2} / 2}{(w-z)^{2}} D_{\epsilon}+\frac{1}{(w-z)^{2}} \epsilon \Theta\left(X^{0}\right)
$$

From this, and making use of general properties of logarithmic operators, listed in the Appendix, we may identify the $C$ operator to be

$$
C_{\epsilon}=\epsilon \Theta_{\epsilon}\left(X^{0}\right)
$$

Note the factor of $\epsilon$. The action of the stress tensor on the operators $C_{\epsilon}$ and $D_{\epsilon}$ reveals [11] that they have the same conformal dimension $\Delta=-\frac{\epsilon^{2}}{2}$ which is negative and vanishes in the limit $\epsilon \rightarrow 0^{+}$(note that this implies that the total dimension of the impulse operator (8), including the $\partial_{n} X^{i}$ factor, is $1-\frac{\epsilon^{2}}{2}$ ). We see that for non-zero $\epsilon$ the impulse operator is relevant in a renormalization-group sense, and the resulting theory is slightly non critical [11, 17].

It is clear from (13) that we cannot work just with the $D_{\epsilon}\left(X^{0}\right)$ operator because $C_{\epsilon}\left(X^{0}\right)$ will necessarily be induced by a scale transformation. Thus, the proper recoil operator is described by

$$
V_{\text {rec }}=\int d \tau\left[y_{i} C_{\epsilon}\left(X^{0}\right) \partial_{n} X^{i}+u_{i} D_{\epsilon}\left(X^{0}\right) \partial_{n} X^{i}\right]
$$

where the coupling constants $y_{i}$ and $u_{i}$ in principle depend on the world-sheet renormalization scale.

In ref. [11] explicit expressions were derived for the one and two point functions of the logarithmic pair $C$ and $D$. For later use we list bellow the result for the two-point functions (concentrating again for brevity on the $X^{0}$ parts):

$$
\begin{aligned}
<C_{\epsilon}(z) C_{\epsilon}(0)> & \sim-\frac{1}{g_{s}} \epsilon^{2} \sqrt{\frac{\pi}{\alpha}} \int_{-\infty}^{\infty} \frac{d q}{\left(q^{2}+\epsilon^{2}\right)} e^{-2 \eta q^{2} \log |z / a|^{2}} \\
& \stackrel{\epsilon \rightarrow 0}{\sim} \frac{1}{g_{s}}\left(0+O\left(\epsilon^{2}\right)\right)
\end{aligned}
$$

with $\alpha \equiv \log |L / a|^{2}$, and $L$ and $a$ denoting the world-sheet infrared and ultraviolet cutoffs respectively, and $\eta=1(-1)$ for Euclidean (Minkowskian) signature of $X^{0}$ in the target space time of the scaffolder. Above we have re-instated the explicit powers of the string coupling constant, $g_{s}$, appropriate for the disc topology. 
In a similar manner one has:

$$
\begin{aligned}
<C_{\epsilon}(z) D_{\epsilon}(0)> & \sim-\frac{\epsilon}{2 g_{s}} \sqrt{\frac{\pi}{\alpha}} \frac{\partial}{\partial \epsilon} \int_{-\infty}^{\infty} \frac{d q}{q^{2}+\epsilon^{2}} e^{-2 \eta q^{2} \log |z / a|^{2}} \\
& \stackrel{\epsilon \rightarrow 0}{\sim} \frac{\pi}{2 g_{s}} \sqrt{\frac{\pi}{\epsilon^{2} \alpha}}\left(1-2 \eta \epsilon^{2} \log |z / a|^{2}\right)
\end{aligned}
$$

Finally the two-point function for $D_{\epsilon}$ is given by

$$
\begin{aligned}
<D_{\epsilon}(z) D_{\epsilon}(0)> & =\frac{1}{\epsilon^{2}}<C_{\epsilon}(z) D_{\epsilon}(0)> \\
& \stackrel{\epsilon \rightarrow 0}{\sim} \frac{\pi}{2 g_{s}} \sqrt{\frac{\pi}{\epsilon^{2} \alpha}}\left(\frac{1}{\epsilon^{2}}-2 \eta \log |z / a|^{2}\right)
\end{aligned}
$$

Performing the limit:

$$
\epsilon \rightarrow 0, \quad \epsilon^{2} \log |L / a|^{2} \sim O(1)
$$

we obtain the canonical two-point correlation functions of logarithmic operators (c.f. Appendix). Notice however that $\langle D D\rangle$ is singular in the limit.

Because the exact value of the numerical constant in (19) is a free parameter we may choose it at will (the difference between different choices can be reabsorbed in the redefinition of the $\log z$ term) and thus establish an unambiguous relation between $\epsilon$, the regularization parameter in a target-space, and $L / a$, which is a world-sheet scale. Choosing as normalization:

$$
\frac{1}{\epsilon^{2}}=\eta \log |L / a|^{2}
$$

and then we get (up to a normalization factor) to zero ${ }^{\text {th }}$ order in $\epsilon^{2}$ :

$$
\begin{aligned}
& <C_{\epsilon}(z) C_{\epsilon}(0)>\sim 0 \\
& <C_{\epsilon}(z) D_{\epsilon}(0)>\sim \frac{1}{2 g_{s}} \\
& <D_{\epsilon}(z) D_{\epsilon}(0)>\sim \frac{1}{2 g_{s}}\left(\log \left|\frac{L}{a}\right|^{2}-2 \eta \log \left|\frac{z}{a}\right|^{2}\right)
\end{aligned}
$$

In the following, as we will consider the euclidean case (for the scaffolding space-time), we set $\eta=1$.

To recapitulate, therefore, the requirement of cancelling anulus infinities in the scaffolder $\sigma$-model with matter (3i) one must include the pair of logarithmic operators $C$ and $D$, which mix under scaling. The full action, which is free, therefore, from conformal 
anomalies up to anulus level reads:

$$
\begin{aligned}
S_{\sigma}= & \int_{\Sigma}\left[\partial X^{\mu} \bar{\partial} X^{\nu} \eta_{\mu \nu}+T(X)\right]+\int d \tau\left[x_{i} \partial_{n} X^{i}+v_{i} X^{0} \partial_{n} X^{i}\right]+ \\
& \int d \tau\left[y_{i} C_{\epsilon}\left(X^{0}\right) \partial_{n} X^{i}+u_{i} D_{\epsilon}\left(X^{0}\right) \partial_{n} X^{i}\right]
\end{aligned}
$$

with $D$ and $C$ given by $(12,14)$.

\section{The Space of Running Coupling Constants as a Phase Space}

In this and the following sections we will discuss how the couplings $y_{i}$ and $u_{i}$ of the operators $C$ and $D$ in (22) can be seen as the phase space of a point particle. From a $\sigma$-model view point we shall consider the action (22) with $T(X)=0, x_{i}=0$ and $v_{i}=0$. According to the discussion above this truncated $\sigma$-model is slightly non-critical, and requires Liouville dressing for consistency. For our purposes we shall treat the Liouville mode as independent from the target time $X^{0}$ of the scaffolder $\sigma$-model. This is due to the fact that we are not interested in the scaffolder dynamics. The resulting Liouville-dressed couplings, then, $y_{i}$ and $u_{i}$ exhibit non-trivial world-sheet scaling. We shall study their behaviour under Liouville renormalization-group flow. Following general properties of non-critical strings [15, 16], then, we shall use the renormalization-group flow as a variable defining temporal evolution in this phase space, in the sense of Galilean invariance.

\subsection{Finite-Size Scaling as Galilean Invariance}

Let us consider a finite size scaling [11]

$$
L \rightarrow L^{\prime}=L e^{t}
$$

Because of the relation between $\epsilon$ and $L$ this transformation will change $\epsilon$

$$
\epsilon^{2} \rightarrow \epsilon^{\prime 2}=\frac{\epsilon^{2}}{1+2 \epsilon^{2} t}
$$

(note that if $\epsilon$ is infinitesimally small then $\epsilon^{\prime}$ is also infinitesimally small for any finite $t$ ) and we can deduce from the scale dependence of the correlation functions (21) that $C_{\epsilon}$ and $D_{\epsilon}$ transform as:

$$
\begin{aligned}
& D_{\epsilon} \rightarrow D_{\epsilon^{\prime}}=D_{\epsilon}+t C_{\epsilon} \\
& C_{\epsilon} \rightarrow C_{\epsilon^{\prime}}=C_{\epsilon}
\end{aligned}
$$


From this transformation one can see that the coupling constants in front of $D_{\epsilon}$ and $C_{\epsilon}$ in the operator defined in (15), i.e. the velocities $u_{i}$ and spatial collective coordinates $y_{i}$ of the brane, must transform like:

$$
u_{i} \rightarrow u_{i} \quad, \quad y_{i} \rightarrow y_{i}+u_{i} t
$$

And this is a Galilei transformation for the $y_{i}$ and $u_{i}$ 's.

We have a first important result, the scale transformation of the two-dimensional theory is a symmetry in the space of coupling constants which coincides with a Galilei transformation.

We can consider this scaling as a renormalization flow on the world sheet of the open string. In fact from (20) and (23) we see that a scale transformation is equivalent to a rescaling of $\epsilon$. The corresponding $\beta$ functions are

$$
\begin{aligned}
\beta^{y_{i}} & =-\frac{\epsilon^{2}}{2} y_{i}+u_{i} \\
\beta^{u_{i}} & =-\frac{\epsilon^{2}}{2} u_{i}
\end{aligned}
$$

where the first part corresponds to the anomalous scaling dimensions of the operators $C$ and $D$ [11]. From (27) it becomes clear that the coupling of the $D$ (momentum) operator in (15) is not exactly marginal as would have been required by the Galilean transformation, were $t$ to be identified as time. There are small $\mathcal{O}\left[\epsilon^{2}\right]$ anomalous dimension terms that spoil this marginality. This can be remedied by redefining the string coupling constant $g_{s}$ so as to absorb such terms. Indeed, it is the coupling [17]

$$
u_{i}^{R} \equiv \frac{u_{i}}{\epsilon}
$$

which is exactly marginal,

$$
d u_{i}^{R} / d t=0
$$

and therefore plays the rôle of a Galilean velocity. This amounts to a re-normalization of $g_{s}$ such that

$$
g_{s}^{R}=\frac{g_{s}}{\epsilon}
$$

is $t$-independent.

The corresponding 'renormalization' of the position

$$
y_{i}^{R} \equiv \frac{y_{i}}{\epsilon}
$$

yields the Galilean equation for (renormalized) velocities:

$$
d y_{i}^{R} / d t=u_{i}^{R}
$$

It is this set of renormalized couplings $y_{i}^{R}, u_{i}^{R}$ that constitute our classical phase space, which may be quantized canonically as we discuss in the subsequent sections. 


\subsection{Helmholtz Conditions and Quantization of Coupling Con- stants}

The above analysis implies that in the presence of the logarithmic operators $C$ and $D$ the D-brane $\sigma$-model exhibits marginal deviations from conformal invariance. It is however known [21] that any non-critical string $\sigma$-model can become conformal by introducing a Liouville mode. The latter may be viewed [15] as a local renormalization scale on a curved world sheet. For our case, this will promote the first order equation (32) to a second order one with respect to the Liouville zero mode. This kind of equation can be derived from a Lagrangian provided a certain set of conditions due to Helmholtz is satisfied [18, 5]. This is necessary and sufficient for canonical quantization.

In fact [22, 15, 19], the most generic renormalization group flow for a $\sigma$-model coupling $\lambda_{i}$, corresponding to a vertex operator $V_{i}$, in Liouville string theory has the form of a friction equation of motion [四]:

$$
\ddot{\lambda}_{i}(\phi)+Q \dot{\lambda}_{i}(\phi)=-\beta^{i}(\lambda)=G^{i j} \frac{\partial}{\partial \lambda_{j}} C(\lambda)
$$

where the dot represents differentiation to Liouville zero mode $\phi, \beta^{i}$ is the flat world-sheet renormalization group $\beta$ function, and $Q^{2}=C[\lambda]-25$ is the central-charge deficit. The quantity $C(\lambda)$ is the Zamolodchikov $C$-function [3]. This function interpolates among conformal field theories according to the $C$-theorem, which for flat world sheets reads:

$$
\frac{\partial C}{\partial t}=-\beta^{i} G_{i j} \beta^{j}
$$

and $G^{i j} \sim<V_{i} V_{j}>$ is the metric in theory space.

In equation (33) we took into account the gradient flow property of the $\beta$-functions:

$$
\partial_{i} C[\lambda]=G_{i j} \beta^{j}
$$

which is an off-shell corollary of the flat-world-sheet $C$-theorem [3]. Equation (33) is characteristic of frictional motion in a potential $C\left(\lambda_{i}\right)$. As known in general such equations cannot be cast in a Lagrangian form. But in the non-critical-string case this is possible due to the non-trivial metric $G_{i j}$. In fact, the situation is entirely similar to the case of inflationary theories [4.

The conditions for the existence of an underlying Lagrangian $L$ [18 whose equations of motion are equivalent (but not necessarily identical) to (33), are equivalent to the existence of a non-singular matrix $w_{i j}$ :

$$
w_{i j}\left(\ddot{\lambda}^{j}+Q \dot{\lambda}^{j}+\beta^{j}\right)=\frac{d}{d t}\left(\frac{\partial L}{\partial \dot{\lambda}_{i}}\right)-\frac{\partial L}{\partial \lambda_{i}}
$$


which obeys the following Helmholtz conditions :

$$
\begin{aligned}
w_{i j} & =w_{j i} \\
\frac{\partial w_{i j}}{\partial \dot{\lambda}^{k}} & =\frac{\partial w_{i k}}{\partial \dot{\lambda}^{j}} \\
\frac{1}{2} \frac{D}{D \phi}\left(w_{i k} \frac{\partial f^{k}}{\partial \dot{\lambda}^{j}}-w_{j k} \frac{\partial f^{k}}{\partial \dot{\lambda}_{i}}\right) & =w_{i k} \frac{\partial f^{k}}{\partial \lambda^{j}}-w_{j k} \frac{\partial f^{k}}{\partial \lambda_{i}} \\
\frac{D}{D \phi} w_{i j} & =-\frac{1}{2} w_{i k} \frac{\partial f^{k}}{\partial \dot{\lambda}^{j}}-\frac{1}{2} w_{j k} \frac{\partial f^{k}}{\partial \dot{\lambda}_{i}}
\end{aligned}
$$

where

$$
f^{i} \equiv-Q \dot{\lambda}^{i}-\beta^{i}(\lambda) \quad ; \quad \frac{D}{D \phi} \equiv \partial_{t}+\dot{\lambda}^{i} \partial_{i}+f^{i} \frac{\partial}{\partial \dot{\lambda}^{i}}
$$

If the conditions (37) are met, then

$$
w_{i j}=\frac{\partial^{2} L}{\partial \dot{\lambda}^{i} \partial \dot{\lambda}^{j}}
$$

The Lagrangian in (39) can be determined up to total derivatives according to [18]:

$$
\begin{aligned}
\mathcal{S} \equiv \int d \phi^{\prime} L & =-\int d \phi^{\prime} \int_{0}^{1} d \kappa \lambda^{i} E_{i}\left(\phi^{\prime}, \kappa \lambda, \kappa \dot{\lambda}, \kappa \ddot{\lambda}\right) \\
E_{i}\left(\phi^{\prime}, \lambda, \dot{\lambda}, \ddot{\lambda}\right) & \equiv w_{i j}\left(\ddot{\lambda}^{j}+Q \dot{\lambda}^{i}+\beta^{i}\right)
\end{aligned}
$$

In the case of non-critical strings one can identify $4 w_{i j}=-G_{i j}$. Near a fixed point, where the variation of $Q$ is small, the action $\mathcal{S}$ becomes 4 , 5]:

$$
\mathcal{S}=\int d \phi^{\prime}\left(-\frac{1}{2} \dot{\lambda}^{i} G_{i j}(\lambda, \phi) \dot{\lambda}^{j}-C[\lambda]+\ldots\right)
$$

with the ... denoting terms that can be removed by a renormalization scheme change.

Within a critical-string (on-shell) approach, the action (40,41) can be considered as an effective action generating the string scattering amplitudes. Here it should be considered as a target-space 'off-shell' action for non-critical strings [15, 4]. From (41) it follows that the canonical momenta $p_{i}$ are given by:

$$
p_{i}=G_{i j} \dot{\lambda}^{j}
$$

Let us now check the validity of the conditions (37). We know that $G_{i j}$ is symmetric, so the first of the Helmholtz conditions (37) is satisfied. The next two conditions hold automatically because of the gradient flow property (35) of the $\beta$ function, and the fact 
that $G_{i j}$ and $C[\lambda]$ are functions of the coordinates $\lambda^{i}$ and not of the conjugate momenta. Finally, the fourth Helmholtz condition provides the condition

$$
\frac{D}{D \phi} G_{i j}=Q G_{i j}
$$

which implies an expanding scale factor for the metric in theory space ('inflation')

$$
G_{i j}[\phi, \lambda(\phi)]=e^{Q \phi} \hat{G}_{i j}[\phi, \lambda(\phi)]
$$

where $\hat{G}_{i j}$ is a Liouville-renormalization-group invariant function. This is exactly the form of the Zamolodchikov metric in Liouville strings [4, 月]. Thus there is indeed an underlying Lagrangian in the non-critical (Liouville) string problem.

This allows canonical quantization, which as we will see in the next section, is induced by higher genus effects [15, 5]:

$$
\left[\lambda^{i}, \lambda^{j}\right]=0 \quad ; \quad\left[p_{i}, p_{j}\right]=0 \quad ; \quad\left[\lambda^{i}, p_{j}\right]=-i \hbar \delta_{j}^{i}
$$

with the commutators being defined in theory space and $p_{i}$ denoting the momentum (42) conjugate to $\lambda^{i}$.

Let us now come to the specific problem of $D$ branes. The pertinent system of flatspace Renormalization-Group flow equations is given by (29,32). Liouville dressing is necessary for the position coupling $y_{i}^{R}$ only, since $u_{i}^{R}$ is exactly marginal. The corresponding central-charge deficit may be found by applying the flat world-sheet Zamolodchikov $C$-theorem (34) for the deformation pertaining to the $y_{i}^{R}$ coupling:

$$
Q^{2}\left[y_{i}^{R}, u_{i}^{R}\right] \propto C\left[t, y_{i}^{R}, u_{i}^{R}\right]-25 \sim-\int_{0}^{t} d t^{\prime} \frac{d y_{i}^{R}}{d t^{\prime}} \epsilon^{2} G_{C C} \frac{d y_{i}^{R}}{d t^{\prime}} \sim-\frac{1}{g_{s}^{R}} \epsilon^{3} t u_{i}^{R} u^{i, R}
$$

where $t$ is the flat world-sheet scale (20) and we used (16). As we discussed above, $t \epsilon^{2} \sim O[1]$, hence the central-charge deficit becomes of order $-\frac{\epsilon}{g_{s}^{R}} u_{i}^{R} u^{R^{i}}$. This choice corresponds to the identification of the logarithm of the size of the world-sheet disc with a (covariant) scale, which is nothing other than the zero mode of the Liouville field $\phi$. However, this identification must be made only at the very end of the computations, and hence at all intermediate steps the Liouville scale should be treated independent of $\epsilon$. Moreover, the exactly marginal coupling $u_{i}^{R}$ is treated, of course, as independent of $d y_{i}{ }^{R} / d t$. It is then straightforward to see that the corresponding Liouville problem satisfies the Helmholtz conditions (37) with $f_{i}=-\dot{y}_{i}^{R} \sqrt{\frac{\epsilon}{g_{s}^{R}} u_{j}^{R} u^{j R}}-u_{i}^{R}$.

Therefore, in our case equation (33) reads:

$$
\ddot{y}^{i R}+\left(\sqrt{\frac{\epsilon}{g_{s}^{R}} u_{j}^{R} u^{j R}}\right) \dot{y}^{i R}=0
$$


Near the fixed point $\epsilon \rightarrow 0$ this becomes the equation of a free particle. Thus, the system of $\sigma$-model couplings $y_{i}^{R}$ can be quantized canonically á la (45). Once we have established a canonical quantization we can then take the flat world-sheet limit, provided we make the above identification of the world-sheet renormalization scale with the area of the disc. This will be assumed in what follows.

The effective action (41), then, simply corresponds to a free phase-space action for a particle of mass $\propto 1 / g_{s}^{R}$ :

$$
\mathcal{S}=-\int d t \frac{1+O[\epsilon]}{2 g_{s}^{R}} u_{i}^{R} u^{i, R}
$$

consistent with the non-interacting brane background at the level of the scaffolder $\sigma$ model. The fact that the mass of the particle is proportional to the inverse coupling constant of the string suggests that there is a natural length scale $\alpha_{s}^{\prime}$ in the problem

$$
\alpha_{s}^{\prime}=\left(g_{s}^{R}\right)^{2} \alpha^{\prime}
$$

where $\alpha^{\prime}$ is the string Regge slope. Such a rescaling is characteristic of soliton strings [23, 6, 5].

We now note that from the point of view of a D-particle (scaffolder) its mass is determined by (10). However in our construction, where the scaffolder is only an auxiliary entity used simply to induce the relevant deformations $C$ and $D$ in a formal manner, there is no such restriction. We can therefore just take $p_{i}=\kappa u_{i}{ }^{R}$ with $\kappa$ an arbitrary scale with dimensions of mass.

Then, the mass of the particle whose action is described by (48) is

$$
M=g_{s}^{R} \kappa^{2}
$$

As we shall show in section 4 this point of view can be supported explicitly by a $\sigma$-model path integral analysis on resummed (pinched) world sheets.

An immediate question arises as to how one can define explicitly the $\hbar$ in theory space. Although the above approach tells us that a canonical quantization may be achieved, however, it does not specify the details of the passage from classical to quantum mechanics. In string theory this can be achieved by a summation over genera on the world-sheet [4. Below we shall review briefly the basic steps by concentrating on the specific case of Dbranes. As we shall argue, $\hbar$ will turn out to be proportional to the square of the string coupling constant. 


\section{$4 \sigma$-model Approach to the Quantization of Phase Space}

The necessity to go beyond genus zero world-sheet surfaces appears quite natural in the framework of the scaffolder $\sigma$-model. Since the effects associated with a change in a conformal field theory background, such as recoil, or in general back-reaction of matter on the structure of space-time etc, are purely stringy, the most natural way of incorporating them in a $\sigma$-model language is to go beyond a fixed genus $\sigma$-model and consider the effects of resummation over world-sheet genera [15, 9, 8]. This is a very difficult procedure to be carried out analytically. However, for our purposes a sufficient analysis, which should describe the situation satisfactorily (at least at a qualitative level), is that of a heavy extended object in target space, which can be treated semi-classically. From a first-quantized point of view, this implies resumming one-loop (anulus) worldsheets. Non-trivial effects arise from degenerate Riemann surfaces, namely from long-thin world-sheet strips or tubes (for the closed-string case) (wormholes) that are attached to a Riemann surface of lower genus (disc in our case).

\subsection{Phase Space and Pinched World Sheets}

We represent the Riemann surface $\Sigma$ as a strip connecting two Riemann surfaces $\Sigma_{1}$ and $\Sigma_{2}$ of lower genus. We take account of the degenerate strip by inserting a complete set of intermediate string states $\mathcal{E}_{\alpha}$ [24]. Then a generic $N$-point correlation function of vertex operators $V_{i}$

$$
A_{N}=\int d m_{\Sigma}\left\langle\prod_{i} \int d^{2} \xi_{i} V_{i}\left(\xi_{i}\right) \otimes(\text { ghosts })\right\rangle_{\Sigma}
$$

is given by the following expression

$$
\begin{array}{r}
A_{N} \sim \sum_{\alpha} \int d q \int d^{2} z_{1} \int d^{2} z_{2} \int d m_{\Sigma_{1} \oplus \Sigma_{2}} \\
\left\langle\prod \prod_{i} \int d^{2} \xi_{i} V_{i}\left(\xi_{i}\right) \mathcal{E}_{\alpha}\left(z_{1}\right) \otimes q^{L_{0}-1} \otimes \mathcal{E}_{\alpha}\left(z_{2}\right) \otimes(\text { ghosts })\right\rangle_{\Sigma_{1} \oplus \Sigma_{2}}
\end{array}
$$

where $q$ is the (real) modular parameter of the strip, $\int d m$ denotes integration over moduli space, and $\mathcal{E}_{\alpha}$ are the complete set of the intermediate states with dimensions $h_{\alpha}$ propagating along the thin strip connecting the world-sheet pieces $\Sigma_{1}$ and $\Sigma_{2}$ (in our case $\Sigma_{1}=\Sigma_{2}$ ). The terms 'ghosts' indicate appropriate insertions of ghost fields, which, although necessary for the target space reparametrization, are of no consequences here. 
One easily observes that logarithmic divergences in (52), may come from states with $h_{\alpha}=0$ [24], in which case one has the infrared divergence at small $q \rightarrow 0$ integral $\int d q / q$. A trivial example of such an operator is the identity, which however does not lead to non-trivial effects, since it carries zero measure in the space of states, and hence it does not contribute to (52). On the other hand, if there are states that are separated by a gap from the continuum of states, i.e. discrete in the space of states, then they bear nontrivial contributions to the sum-over-states and lead to divergences in the amplitude (52) [8, 9, 15, 19, 5].

The logarithmic states we discussed earlier are precisely these states. There are various types of divergences associated with such states, as discussed in ref. [9]. First of all, there are leading divergent terms in (52) arising due to the mixing between the states corresponding to the logarithmic operators $C$ and $D$ :

$$
\begin{aligned}
\operatorname{Diverg}[\mathrm{STRIP}] & \sim g_{s} \int_{q \sim \delta \rightarrow 0} \frac{d q}{q} \ln q \int d^{2} z_{1} D\left(z_{1}\right) \int d^{2} z_{2} C\left(z_{2}\right) \\
& \sim g_{s}\left(\ln ^{2} \delta\right) \int d^{2} z_{1} D\left(z_{1}\right) \int d^{2} z_{2} C\left(z_{2}\right)
\end{aligned}
$$

This gives the leading singularity $\ln ^{2} \delta$.

The effects of a dilute gas of wormholes on the sphere are assumed to exponentiate this bilocal operator and one can obtain a change in the world-sheet action [8, 9]

$$
\Delta S \sim g_{s}\left(\ln ^{2} \delta\right) \int d^{2} z_{1} D\left(z_{1}\right) \int d^{2} z_{2} C\left(z_{2}\right)
$$

This bilocal term can be written as a local world-sheet effective action term, if one employs the well-known trick of wormhole calculus [25] by writing

$$
e^{\Delta S^{C D}} \propto \int d \gamma_{C} d \gamma_{D} \exp \left[-\frac{1}{4 g_{s} \ln ^{2} \delta} G^{m n} \gamma_{m} \gamma_{n}+\gamma_{C} \int d^{2} z C+\gamma_{D} \int d^{2} z D\right]
$$

In this way the bilocal operator can be represented as a local deformation on the worldsheet of the string but with deformation couplings $\gamma_{m}$, where $m=C$ or $D$ and $G^{m n}$ is an off-diagonal metric in coupling-constant space, necessary to reproduce the initial bilocal operator. The exponentiation of thin handles had been assumed above. The off-diagonal metric in theory space includes all the appropriate normalization factors $\mathcal{N}_{m}$, for the zero mode states. Such factors are essentially the inverse of the $C D$ two-point function of the operators $C$ and $D$ given in (17), which is finite 11].

Besides the leading divergent terms in (52) there will be terms with $\ln \delta$ behaviour, corresponding to

$$
\begin{aligned}
\Delta S^{D} & \equiv g_{s} \ln \delta \int d^{2} z_{1} D\left(z_{1}\right) \int d^{2} z_{2} D\left(z_{2}\right) \\
\Delta S^{C} & \equiv g_{s} \ln \delta \int d^{2} z_{1} C\left(z_{1}\right) \int d^{2} z_{2} C\left(z_{2}\right)
\end{aligned}
$$


Assuming their exponentiation, one may use new wormhole parameters $\alpha_{C}$ and $\alpha_{D}$ to represent such counterterms in the world-sheet action as above. The only difference now is that the distribution functions for the wormhole parameters associated with the subleading $\ln \delta$ divergences are ordinary Gaussian associated with symmetric metrics $G_{C C}, G_{D D}$ in coupling-constant space:

$$
e^{\Delta S^{D}+\Delta S^{C}}=\int d \alpha_{C} d \alpha_{D} e^{-\frac{\alpha_{c}^{2} G C C}{\Gamma_{C}^{2}}} e^{-\frac{\alpha_{D}^{2} G^{D D}}{\Gamma_{D}^{2}}} e^{-\alpha_{C} \int d^{2} z C_{\epsilon}-\alpha_{D} \int d^{2} z D_{\epsilon}}
$$

where the widths $\Gamma_{C}^{2}, \Gamma_{D}^{2}$ are given by:

$$
\Gamma_{C}^{2} \sim 4 g_{s} \ln \delta, \quad \Gamma_{D}^{2} \sim 4 g_{s} \ln \delta
$$

and the diagonal metrics elements contain now zero-mode-state normalization factors which are given by the absolute value of the inverse of the $C C$ (16) and $D D(18)$ twopoint functions

$$
\left|G^{C C}\right| \sim \frac{1}{\mathcal{N}_{C}^{2}} \sim \frac{\epsilon^{2}}{g_{s}} \quad G^{D D} \sim \frac{1}{\mathcal{N}_{D}^{2}} \sim \frac{1}{2 g_{s} \epsilon^{2}}
$$

From this analysis it becomes clear that the above-described pinched-anulus-corrected string matrix elements are divergent for $\epsilon^{2} \rightarrow 0^{+}$. From a two-dimensional (world-sheet) view-point one should either cancel or absorb the divergences in coupling constants. If all the divergences could be canceled, then the $\sigma$-model would be conformally invariant, whilst absorption of the divergent contributions to $\sigma$-model couplings would result in departures from criticality 15.

\subsubsection{Leading Divergences}

First, let us concentrate on the term (55). Following ref. [8], we consider the propagation of two matter (closed-string) tachyon states $V_{1,2}$ in the background of (55) at tree level. In such a computation the effects of the $C$-operator are subleading and can be ignored. The result is:

$$
\mathcal{A}_{C D} \equiv<V_{1} V_{2} e^{\Delta S^{C D}}>_{*} \sim \int d \gamma_{C} d \gamma_{D} \exp \left[-\frac{1}{4 g_{s} \ln ^{2} \delta} \gamma_{m} G^{m n} \gamma_{n}+\gamma_{D} \int d^{2} z D+\ldots\right] V_{1} V_{2}
$$

where the $\langle\ldots\rangle_{*}$ denote averages with respect to a conformal $\sigma$-model action on the disc, and ... represent subleading terms.

The scaling property (25) must be taken into account. Under scaling on the world-sheet the $C$ operator emerges from $D$ due to mixing (see Appendix), with a scale-dependent coefficient $u_{D} t$. This will contribute to the scaling infinities we are considering here. 
The $D$ operator insertion in (60) needs to be normal-ordered in the disc. Normal ordering here amounts to subtracting scaling infinities originating from divergent contributions of the composite operator $D$ as $\epsilon \rightarrow 0^{+}$. To determine these infinities we first note that due to the $X^{i}$ parts of the operators $D$, their one-point function on the disc, computed with respect to the free $\sigma$-model action, may be written as

$$
<e^{-\int d \tau D}>_{*} \sim e^{\frac{1}{2} \int_{\partial \Sigma} \int_{\partial \Sigma} d \tau d \tau^{\prime}<D(\tau) D\left(\tau^{\prime}\right)>_{*}}
$$

As a consequence of (18) and (25), there are leading (scaling) divergences for $\epsilon \rightarrow 0^{+}$, which behave as $\frac{1}{4 g_{s}} t^{2} \gamma_{D}^{2} \epsilon^{2} \sim \frac{1}{2 g_{s}} \gamma_{D}^{2} t$, using the normalization (20) between $t$ and $\epsilon$ [11. Hence, normal ordering of the $D$ operator amounts to adding a term $-\frac{1}{2 g_{s}} t \gamma_{D}^{2}$ in the exponent of (60) in order to subtract such divergences.

Let us now introduce a complete set of states $\mid \phi_{\alpha}>$ in the two-point function of string matter on the disc:

$$
<V_{1} V_{2}>_{*}=\sum_{\alpha} \mathcal{N}_{\alpha}^{2}<V_{1}\left|\phi \alpha>_{*}<\phi \alpha\right| V_{2}>_{*}
$$

where $\mathcal{N}_{\alpha}^{2}$ is a normalization factor for states. Taking into account (59), and the discussion above, according to which the effects of the $C$ operator are also included in the $D$ under scaling (25) [11, one observes that the leading divergent contributions in (62) are of the form

$$
<V_{1} V_{2}>_{*} \sim-g_{s} t<V_{1}|C><C| V_{2}>\text { +subleading terms }
$$

We now remark that the operator $C$ plays the rôle of the Goldstone modes for translation of the collective coordinates $X^{i}$ of the scaffolder, and as such one may apply the corresponding Ward identity in the $\sigma$-model path-integral to represent the action of $C$ on a physical state by $-\partial / \partial X^{i}[8,5]$. Thus, to this order in the string-coupling constant, the result may exponentiate to yield

$$
<V_{1} \mid V_{2}>_{*} \sim \exp \left(-\frac{1}{2} g_{s} t\left(\frac{\partial}{\partial X^{i}}\right)^{2}\right)
$$

where we used the on-shell condition $V_{j}\left(\frac{\partial^{2}}{\partial X^{i^{2}}} V_{i}\right)=0$.

Then, (60) may be expressed as

$$
\begin{aligned}
\mathcal{A}_{C D} & \sim \int d \gamma_{D} d \gamma_{C} \exp \left[-\frac{1}{4 g_{s} \ln ^{2} \delta} \gamma_{m} G^{m n} \gamma_{n}-\frac{1}{2 g_{s}} t \gamma_{D}^{2}+t \gamma_{D}^{i} \frac{\partial}{\partial X^{i}}-\frac{1}{2} g_{s} t \frac{\partial}{\partial X^{i}} \frac{\partial}{\partial X^{i}}+\ldots\right] V_{1} V_{2} \\
& =\int d \gamma_{D} d \gamma_{C} \exp \left[-\frac{1}{4 g_{s} \ln ^{2} \delta} \gamma_{m} G^{m n} \gamma_{n}-\frac{t}{2}\left(\frac{\gamma_{D}^{i}}{\sqrt{g_{s}}}-\sqrt{g_{s}} \frac{\partial}{\partial X^{i}}\right)^{2}+\ldots\right] V_{1} V_{2}
\end{aligned}
$$


with the ... denoting finite terms. From (65) it is evident that if one takes the limit $t \rightarrow \infty$ before the $\gamma_{D, C}$ integration, then one obtains a $\delta$-function factor

$$
\delta^{(d)}\left(\frac{\left(\gamma_{D}\right)_{i}}{\sqrt{g_{s}}}-\sqrt{g_{s}}\left(k_{1}+k_{2}\right)_{i}\right)
$$

where $d$ denotes the spatial dimension of the scaffolder $\sigma$-model, and $\left(k_{1,2}\right)_{i}$ are the momenta of the matter closed string states. From (66) one obtains

$$
u_{i} \sim g_{s}\left(k_{1}+k_{2}\right)_{i}
$$

This formula was mentioned earlier (10), and is consistent with the BPS mass of the stringy soliton $M_{s} \propto \frac{1}{g_{s}}[8]$. The above procedure guarantees conformal invariance of the $\sigma$-model, as far as leading divergences are concerned. This is the same result as the one obtained in ref. [8], but the procedure followed here is formally different.

For our purposes in this work we shall reverse the logic, and consider a $\sigma$-model action (22) with a tree-level coupling $u_{i}$ given by (67), but opposite in sign. The above analysis, then, shows that resumming pinched genera in such a $\sigma$-model, leads -in the limit $\epsilon \rightarrow 0$ - to a cancellation of the classical (tree-level) coupling constant $u_{i}$. As we shall show next, the remaining subleading divergences yield fluctuations of the phase-space couplings around their classical values. This is the basis of our construction of a quantum phase space, and it will be assumed in what follows.

\subsubsection{Subleading Divergences}

We now proceed to treat the subleading divergences of $O[\sqrt{\ln \delta}]$, associated with (57). To this order the partition function of the $\sigma$-model on the resummed pinched topologies (RPT) assumes the form:

$$
\begin{aligned}
Z= & \int D X d \alpha_{m} \exp \left[-\left\{S^{*}+y_{i} \int_{\partial \Sigma} \epsilon \Theta_{\epsilon}\left(X^{0}\right) \partial_{n} X^{i}+u_{i} \int_{\partial \Sigma} X^{0} \partial_{n} X^{i} \Theta_{\epsilon}\left(X^{0}\right)+\right.\right. \\
& \left.\left.\alpha_{C} \sqrt{\ln \delta} \int_{\partial \Sigma} \Theta\left(X^{0}\right) \partial_{n} X^{i}+\alpha_{D} \sqrt{\ln \delta} \int_{\partial \Sigma} X^{0} \Theta\left(X^{0}\right) \partial_{n} X^{i}+\frac{1}{4 g_{s}} G^{m n} \alpha_{m} \alpha_{n}\right\}\right]
\end{aligned}
$$

where $S^{*}$ is the standard free $\sigma$-model action on the 'bulk' of the world sheet?.

From (68) it, then, becomes clear that the effect of the operators $C$ and $D$ is simply to induce quantum fluctuations on $y_{i}$ and $u_{i}$. Indeed by redefining

$$
y_{i}+\alpha_{C} \sqrt{\ln \delta} \rightarrow \hat{y}_{i}, \quad u_{i}+\alpha_{D} \sqrt{\ln \delta} \rightarrow \hat{u}_{i}
$$

\footnotetext{
${ }^{6}$ Note that the normalization of $\alpha_{C}$ and $\alpha_{D}$ has been changed by $\sqrt{\ln \delta}$ as compared to (57), to facilitate the comparison with the tree-level result.
} 
and taking into account that the momentum $p_{i}$ of the 0 brane is $\propto u_{i} / g_{s}$, we can rewrite (68) as a 'quantum' target phase-space path integral :

$$
Z \sim \int d \hat{y}_{i} d \hat{p}_{i} \frac{1}{g_{s} \ln \delta} e^{-\frac{\left(\hat{q}_{m}-q_{m}\right) G^{m n}\left(\hat{q}_{n}-q_{n}\right)}{g_{s} \ln \delta}} \int D X e^{-S^{*}-\int \hat{q}_{m} V_{m}(X)}
$$

where $q_{m} \equiv\left\{y_{i}, g_{s} p_{i}\right\}, V_{m} \equiv\{C, D\}$.

We first examine the Gaussian exponential factors in (70). As we shall argue these are phase-space distribution functions for the fluctuating collective coordinates and momenta of the 0-brane. Thus the effect of resumming (pinched) world-sheet surfaces is to induce a statistical gaussian spread of the couplings. This is the essence of our quantization procedure.

To see this let us first concentrate on the velocity distribution function

$$
e^{-\frac{\left(\hat{u}_{i}-u_{i}\right)^{2}}{g_{S}^{2} \epsilon^{2} \ln \delta}} \equiv e^{-\frac{\left(\delta u_{i}^{(q)}\right)^{2}}{\Gamma_{D}^{2}}}
$$

with

$$
\Gamma_{D}^{2} \sim\left(g_{s}^{R}\right)^{2} \epsilon^{2} \ln \delta
$$

and $\delta u_{i}^{(q)} \equiv \frac{\hat{u}_{i}-u_{i}}{\epsilon}$ denoting the quantum fluctuations of the velocity. We absorbed the $\epsilon^{2}$ dependences in a renormalization of the velocity operators, and hence of $g_{s}$ (see eqns. (28) and (30)). This way the fluctuations of the velocity maintain their exactly marginal character. Indeed, from (70) it becomes evident that the renormalization (30), sought for in the previous section, is a consequence of the summation over pinched handles. Indeed, by making the identification of the infinities due to $\ln \delta, \delta \rightarrow 0$, with world-sheet scaling infinities [26], we observe that the $\alpha_{D}^{i} / \epsilon$ couplings may be marginal if we require that $\alpha_{D}$ behaves like the classical $u_{i}$ under renormalization, i.e.

$$
\frac{d}{d t} \alpha_{D}^{i}=-\frac{1}{2 t} \alpha_{D}^{i}
$$

from which

$$
\delta u_{i}^{(q)} \equiv \alpha_{i D} / \epsilon: \beta_{i}^{\delta u} \equiv \frac{d}{d t} \delta u_{i}^{q}=0
$$

may be considered as an exactly marginal fluctuation of (quantum) velocity operators.

Repeating the reasoning above for the distribution of the $C$ operator we find that its width $\Gamma_{C}^{2}$ behaves as

$$
\Gamma_{C}^{2} \sim\left(g_{s}^{R}\right)^{2} \frac{1}{\epsilon^{2}} \ln \delta \quad t \rightarrow \infty
$$

where, as before, the fluctuations for the position are defined by $\delta y^{i,(q)} \equiv \frac{\hat{y}_{i}-y_{i}}{\epsilon}$. 
In the same spirit, by requiring that $\alpha_{C i}$ behaves like the classical coordinate coupling $y_{i}$,

$$
\frac{d}{d t} \alpha_{C}^{i}=-\frac{1}{2 t} \alpha_{C}^{i}+\alpha_{D}^{i}
$$

one observes that the quantity:

$$
\delta y^{i,(q)} \equiv \alpha_{C}^{i} / \epsilon: \beta^{\delta y} \equiv \frac{d}{d t}\left(\delta y^{i,(q)}\right)=\delta u^{i,(q)}
$$

plays the rôle of a position quantum fluctuation.

It is those (renormalized) quantities $\delta y^{(q)}$ and $\delta u^{(q)}$ that satisfy the canonical commutation relations (45) in theory space, from which the quantization of phase space will emerge consistently, as we show in the next section. For the moment we note that a closer look at the widths $(72,75)$ of the gaussian fluctuations associated with the above quantities reveals an 'uncertainty' relation:

$$
\Gamma_{D}^{2} \Gamma_{C}^{2} \sim g_{R}^{2} \ln ^{2} \delta
$$

From this relation, read as a Heisenberg uncertainty relation, one can infer an upper bound in the Planck's constant in our phase space, proportional to $g_{R}^{2}$. At the level we are presently working, it suffices to view the right hand side of (78) of order of the square of Planck's constant. Taking into account that the operator $D$ refers to velocities and not momenta in target space, one may, then, infer from (78) a minimum uncertainty in phase space associated with Gaussian fluctuations, with

$$
\hbar_{R} \sim g_{R}^{2} \kappa \ln \delta
$$

where the mass scale $\kappa$ was introduced in (79), and arises from the interpretation of $u_{i}$ as a velocity operator in a phase space with momenta $p_{i} \equiv \kappa u_{i}$. Recalling (50) one observes that the particle mass $M=\kappa^{2} g_{s}$ is then independent of the scaffolder soliton mass $1 /\left(g_{s} \sqrt{\alpha^{\prime}}\right)$. We also observe that the subleading infinities associated with thin strips may be absorbed in a redefinition of the string scale $\alpha_{s}^{\prime}(49)$ in order for the right-handside of (79) to be scale independent. With this in mind one then obtains a definition of Planck's constant in this problem proportional to the (string-loop-renormalized) string coupling constant円.

\footnotetext{
${ }^{7}$ We remark that in the case where $X^{0}$ is identified with the Liouville zero-mode $\phi$ [5, 17 one obtains a similar expression for $\hbar$. The physical interpretation, however, is different. One has a stochastic dynamics for the phase-space evolution [17. In such case the width associated with the distribution of the $D$ operator $(72)$ is still scale independent, whilst that of the $C$ operator acquires a stochastic form $\Gamma_{C}^{2} \sim g_{R, \delta}^{2} \phi$ with $g_{R, \delta} \equiv g_{R} \ln \delta$. The associated distribution function may be interpreted [17] as a Gaussian wave-packet of a free D particle, spreading in Euclidean target time $\phi$ (i.e. diffusion) with spread $(\hbar / m) \phi$ with $\hbar / m$ as in (79).
} 


\subsection{Phase-Space Dynamics}

Finally we come to the integral over the $X$ fields in (70), which will yield the target-space effective action of the scaffolder, depending on the (quantum) backgrounds $\hat{q}_{i}$. In the case at hand this will yield the dynamics in phase space. There are two ways one can proceed, which depend on whether one identifies the renormalization scale with the target time $X^{0}$ of the scaffolder or not. In this latter case, the $X$ integration yields an effective action of a non-critical string theory. According to the analysis in ref. [27, 15, 5] this is nothing other than the Zamolodchikov $C$ function for the specific problem. Using the $C$-theorem [3], then, one has:

$$
S_{e f f} \sim C\left[\hat{y}^{R}, \hat{u}^{R}, t\right] \sim-\int_{0}^{t} d t^{\prime} \epsilon^{2} \beta^{y} G_{C C} \beta^{y}=-\int_{0}^{t} d t^{\prime} \frac{1}{g_{R}} \epsilon\left(u_{i}^{R}\right)^{2}
$$

due to $(16),(30),(29),(32)$. This is the action of a free particle moving with constant velocity $u_{i}^{R}$, and thus the situation is in agreement with (48).

For completeness let us now compare this result with the one obtained under the identification of $X^{0}$ with the Liouville scale $\phi$, which is not necessary in this paper. In such a case the scaffolder dynamics must be taken into account [5, 17]. The $X$ integration may then be performed in a standard fashion in D-brane formalism, by noting the equivalence of the D-brane background to that of an open string propagating in the background of imaginary gauge potentials $A_{M}$ [6]. This yields the Born-Infeld action, $\int d X^{0} d^{9} X^{i} \sqrt{1-\left|F_{M N}^{2}(A)\right|}$, as the effective action obtained from $X$ integration.

In our case, the particular form of the action (68.70), and eqs. (28, 29) suggest that the pertinent gauge potential is that of a space-constant 'electric' field:

$$
A_{i}=-i \epsilon\left(\hat{y}_{i}+\hat{u}_{i}^{R} X^{0}\right) \Theta_{\epsilon}\left(X^{0}\right)
$$

However, unlike the standard D-brane result [6], here the electric field is singular at $X^{0}=0$ [17]:

$$
i F_{0 i} \equiv i E=\epsilon \hat{u}_{i}^{R} \Theta_{\epsilon}\left(X^{0}\right)+\epsilon\left(\hat{y}_{i}+\hat{u}_{i}^{R} X^{0}\right) \delta_{\epsilon}\left(X^{0}\right)
$$

Were it not for the $\delta\left(X^{0}\right)$ term, the situation would be similar to the free nonrelativistic particle discussed in this work. Indeed, from a Liouville renormalization point of view, such infinities may be interpreted as infrared on the world-sheet, since they correspond to the case where the size of the world sheet has shrunk to the order of the ultraviolet cut-off. One might, then, think of subtracting the $X^{0}=0$ (scaling) infinities from (82) by some regularization procedure. Such terms are not relevant for large-size world sheets as the ones used in the present work. Then, the pertinent effective action 
reads:

$$
\int D X e^{-S^{*}-\int \hat{q}_{m} V_{m}(X)}=e^{-S_{e f f}[\hat{q}]} \quad ; \quad S_{e f f} \propto \frac{1}{g_{s}} \int_{X^{0}>0} d X^{0} d^{9} X^{i} \sqrt{1-\epsilon^{2} u_{i}^{R} u^{i, R}}
$$

and this would lead to the existence of an upper bound in velocities of order $\frac{1}{\epsilon}$. This bound is a consequence of the existence of a critical field in the Born-Infeld Lagrangian [6]. In our case the critical velocity appears to be infinite as $\epsilon \rightarrow 0^{+}$. This is consistent with the non-relativistic nature of the slowly-moving $D$ brane we are employing here. Upon expanding $S_{\text {eff }}$ in (83) in powers of $\epsilon^{2}$, and keeping the non-trivial leading terms as $\epsilon \rightarrow 0^{+}$ one obtains (for $X^{0}>0$ ) the action of a free particle moving with constant velocity $u_{i}^{R}$, consistent with the result (48).

Unfortunately the above procedure breaks down near $X^{0}=0$, i.e. for very small world sheets, which have to be taken into account if one identifies the Liouville scale $\phi$ with $X^{0}$. In such cases the Born-Infeld Lagrangian exhibits non-trivial mixing between phase-space coordinates $\hat{y}$ and $u^{R}$, inducing singular interactions at $X^{0}=0$, which have been argued in ref. [17 to be responsible for decoherence. This makes an important difference from the treatment in this work, where the Liouville field is taken independent from the scaffolder time $X^{0}$.

\section{Commutators and Uncertainty Principle}

According to ref. [4] and the discussion in sub-section 3.2, summation over world-sheet sheet genera implies a canonical quantization in theory space between the couplings $\left\{\lambda^{i}\right\}$ and the conjugate momenta defined in (42):

$$
\left[\lambda^{i}, G_{m j} \beta^{j}\right]=-i \hbar \delta_{m}^{i}
$$

From the Helmholtz conditions (37) we know that the quantity $\hbar$ is just a constant in the sense that it does not depend on $\lambda$ or the canonical momenta. It may depend on the renormalization-group scale.

The operator prescription of ref. [4] requires the replacement of classical couplings $\lambda^{i}$ by quantum operators in the expressions for $G_{i j}$ and $\beta^{i}$, and the quantum ordering prescription followed is the one that leads to a stochastic quantum diffusion equation [15, 28. Fortunately, for the $G_{i j}$ and to the order of $\epsilon$ we are considering, operator ordering issues will not arise.

In the D-brane $\sigma$-model, perturbed by the boundary recoil operators (15), the couplings are $y_{i}$ and $u_{i}=g_{s} p_{i}$, and the non-vanishing Zamolodchikov metric components 
are $G_{C C} \sim<C C>, G_{D D} \sim<D D>$ and $G_{D C}=G_{C D} \sim<C D>$, given to leading order by the two-point functions (16),(18) and (17) respectively. Higher-order corrections, in a (perturbative) expansion over $\sigma$-model coupling constants, are incorporated in our approach by considering vacuum expectation values $\langle\ldots\rangle$ with respect to an effective $\sigma$-model on the disc, deformed by the operators $C$ and $D$, but with the respective couplings representing the quantum fluctuations $\delta \hat{y}_{i}=\hat{y}_{i}-y_{i}, \delta \hat{p}_{i} \equiv \hat{p}_{i}-p_{i}$ around the classical phase space. As mentioned in section 4, this follows from the requirement of cancellation of the leading divergences in the pinched-world-sheet approximation.

In the D-brane case, the pertinent operators are given by the fluctuations (74) and (77). For the commutator of the $y_{i}$ coupling we have to leading order in $\epsilon^{2}$ :

$$
-i \hbar \delta_{m}^{i}=\left[\delta y^{i, q}, G_{C C} \beta^{\delta y^{m}}\right]=\frac{\epsilon^{2}}{2}\left[\hat{y}_{i}, \hat{p}_{m}\right]
$$

From which

$$
\left[\hat{y}_{i}, \hat{p}_{m}\right]=-i \frac{2 \hbar}{\epsilon^{2}} \equiv \hbar_{R} \quad d \hbar_{R} / d t=0
$$

where $\hbar_{R}$ is defined in (79) and is exactly marginal. The necessity for the marginal character of $\hbar_{R}$ can be seen by deriving equation (85) with respect to $t$, and taking (74) and (77) into account. The constant $\hbar_{R}$ plays the rôle of Planck's constant in our phase space.

To discover stringy effects, one should evaluate $G_{C C}$ not in the free action but in the presence of $C$ and $D$ deformations. In a perturbative expansion in the couplings of the above deformation, one can bring down one more power of the couplings. An analysis similar to the one in ref. [11], then, shows that the dominant contributions come from the $D$ deformations, and are of order $-<C C D>g_{s}^{R} \epsilon^{2} \delta \hat{p}_{i} \sim-\epsilon^{2}\left(\delta \hat{p}_{i}\right)$, where $\delta \hat{p}_{i}=\hat{p}_{i}-p_{i}$ denotes the quantum fluctuations around a classical momentum. The sub-dominant $C$ perturbation may also be easily incorporated by adding a term $-<C C C>\epsilon \delta \hat{y} \sim \frac{\epsilon^{3}}{g_{s}^{R}}(\delta \hat{y})$ in the $G_{C C}$ metric. Thus, proceeding as above, the next-to-leading order coupling-constant corrections to the commutator (86) read:

$$
\left[\hat{y}_{i}, \hat{p}_{m}\right] \sim-i \hbar_{R} \delta_{m}^{i}\left(1-\frac{\epsilon}{g_{s}^{R}} \delta \hat{y}_{m}-2 g_{s}^{R}\left(\delta \hat{p}_{m}\right)+\ldots\right)^{-1}
$$

We now notice that the presence of $(\delta \hat{p})$ - and $(\delta \hat{y})$ - dependent denominators in the commutators (87) implies stringy corrections to the uncertainty. To this end let us first take into account the Gaussian form (70),(57) of the distribution functions of the fluctuations $\delta \hat{y}$ and $\delta \hat{p}$, which implies that any average of linear functions of the respective commutators vanish. 
Thus, from a general property of self-adjoint operators, such as $\hat{y}$ and $\hat{p}$, one can derive from (87) the resulting uncertainty:

$$
\Delta y \Delta p \geq \frac{\hbar_{R}}{2}\left|<\frac{1}{1-\frac{\epsilon}{g_{s}^{R}} \delta \hat{y}-2 g_{s}^{R} \delta \hat{p}+\ldots}>\right| \sim \frac{\hbar_{R}}{2}\left(1+\frac{\epsilon^{2}}{\left(g_{s}^{R}\right)^{2}} \Delta y^{2}+O\left(\alpha_{s}^{\prime}\right) \Delta p^{2}+\ldots\right)
$$

reinstating dimensions with respect to the new D-brane scale (49) $\alpha_{s}^{\prime}=\left(g_{s}^{R}\right)^{2} \alpha^{\prime}$, depending on the string coupling constant. For weakly coupled strings $g_{s}^{R}<<1$ this is smaller than the standard Regge scale. In the limit $\epsilon \rightarrow 0$ the uncertainty (88) is of the form of the string uncertainty principle [20].

This result is in agreement with the Heisenberg-Microscope approach to the uncertainty principle for D-branes obtained in [11]. We also remark that in the context of non-critical strings analogous uncertainty relations lead to measurability bounds for distances, consistent with quantum decoherence [29, 17].

\section{Conclusions and Discussion}

In this paper we have argued that the couplings of the vertex operators describing interaction of a D-brane with string matter, can give rise to a quantum phase space. We did so in the simplest possible case, the lowest dimensional soliton (a 0-brane) in a flat background and ignoring supersymmetry. Moreover we resummed only pinched topologies of the world-sheet. We found that the quantum particle described this way is a free nonrelativistic particle. Less simple solitons and backgrounds should give rise to other (more realistic) structures.

We only considered single-brane backgrounds. Multiple D-branes and possible bound states among them or among strings and D-branes have been ignored. Such structures can also be represented in a conformally invariant way, as argued in ref. [30], at least in flat target-space backgrounds.

In [30] the case of $n$ parallel Dirichlet $p$-branes has been considered in a flat target space. We recall [6] that the D-brane operator (11) or (15) describes transverse excitations of the brane. The collective coordinates $Y_{i}\left(X^{0}, \ldots X^{p}\right)$ may be viewed [30, 7] as scalar fields in the world-volume of the brane $\left\{X^{0}, \ldots X^{p}\right\}$. On the other hand, the longitudinal excitations

$$
A_{I}\left(X^{0}, \ldots X^{p}\right) \partial_{\tau} X^{I} \quad I=0, \ldots, p
$$

are associated with gauge fields on this world volume. For a single brane the gauge group is $U(1)$. According to the analysis of ref. [30], $n$ identical $p$-branes on top of each 
other are described by an effective low-energy field theory on the world volume, with a world-volume $U(n)$ gauge field and $9-p$ scalar fields in the adjoint representation, representing the collective coordinates $X^{i}$. The effective field theory on the world volume of the D-brane is defined, then, as the dimensional reduction of the ten-dimensional supersymmetric Yang-Mills field theory with gauge group $U(n)$. The bosonic part of the effective scalar potential of such a theory is given by

$$
V=\frac{T^{2}}{2} \sum_{i, j=p+1}^{9} \operatorname{Tr}\left[X^{i}, X^{j}\right]^{2}
$$

where $T \propto\left(\alpha^{\prime}\right)^{-1}$ is the D-brane tension, and the trace is over the adjoint representation of $U(n)$.

In the above description the configuration space of the 'collective coordinates' of the system of identical $n$ D-branes consists of effectively noncommuting matrices. When the D-branes are far apart, a classical vacuum solution, with unbroken supersymmetry, minimizing the potential $V=0$ in (90), is the dominant configuration, and one obtains a set of commuting $X^{i}$. In that case the matrices $X^{i}$ can be simultaneously diagonalized [30], with each diagonal entry representing the target-space location of the $k$-th brane, $k=1, \ldots, n$.

At short distances things are by no means simple. When the branes are very close to each other, closer than the scale $\sqrt{\alpha_{s}^{\prime}}$, massive excitations in the spectrum of the $U(n)$ gauge theory must be taken into account. In ref. [30] it was argued that in that case, one should consider the full quantum theory of the $U(n)$ field theory on the world volume, and it might be that states with broken supersymmetry $\left(\left[X^{i}, X^{j}\right] \neq 0\right)$ might arise. If this were the case, this would imply that at very short distances the stringy space time would have a non-commutative structure.

From the results of ref. [5], and the present work, then, one might speculate that, in such a case, summing over world-sheet topologies of the underlying $\sigma$-model might lead to a proper quantization of a noncommutative space-time. Consequently, following the spirit of refs. [31, 32, 33], one could arrive at new forms of uncertainty relations involving only the coordinates of space time, which would be up and above the phase-space uncertainty relation (88).

At present there is no known example of such a situation within the flat-space D-brane theory. We expect that curved target-space backgrounds in string theory play an important rôle in such issues. We base this expectation on our experience with two-dimensional $\sigma$-model supersymmetric field theories [15, 34]. Indeed, the $N=2$ supersymmetric $\sigma$ model on a $S L(2, R) / U(1)$ target manifold, which from a target-space-time point of view describes physics near the singularity of a black hole in string theory [35, 15], admits world-sheet instantons in its spectrum [34]. These are responsible for a breaking of the 
world-sheet supersymmetry in the sense of a 'false' vacuum, characterized by a non-zero vacuum energy. The world-sheet conformal invariance of the model is broken [34, 15]. From a non-critical string point of view, therefore, this situation arguably describes (unstable) vacua of a quantum string theory which should be taken into account.

One can imagine a similar situation happening in our world-volume theory: some non-trivial metric configurations for the collective coordinates of the (quantum) D-branes produce a dynamical breaking of supersymmetry, via some world-brane non-perturbative effects. Probably, also in this case, non-criticality plays a rôle analogous to the one played in the present paper, as a result of the incompatibility of the Dirichlet boundary conditions with world-sheet conformal invariance [6, 36].

Needless to say, much more work is needed before even tentative conclusions are reached as regards the quantum structure of space-time. Nevertheless, we believe that the present work provides compelling evidence for the importance of solitonic string backgrounds in the structure of quantum physics and the structure of space time.

\section{Appendix: Logarithmic Operators}

In this appendix we discuss the appearance of pairs of logarithmic operators in the correlation functions of conformal field theories. We will limit ourselves to a sketchy introduction

of them in the theories of interest to us. For more details we refer the interested reader to the literature, and in particular to [12, 13, 37, 9, 11].

It is well known that in general, hypergeometic equations of the kind:

$$
x(1-x) \frac{d^{2} \mathcal{F}}{d x^{2}}+[c-(a+b+1) x] \frac{d \mathcal{F}}{d x}-a b \mathcal{F}=0
$$

admit two independent solutions (see for example [38, 39])

$$
\mathcal{F}_{1}=F(a, b, c ; x), \quad \mathcal{F}_{2}=x^{1-c} F(a-c+1, b-c+1,2-c ; x)
$$

where $F(a, b, c ; x)$ is the hypergeometric function.

However in the case in which $c$ is an integer the two solutions would be degenerate, and they are, in the case of $c>1$ :

$$
\begin{aligned}
& \mathcal{F}_{1}=F(a, b, 1+m ; x), \\
& \mathcal{F}_{2}=\ln x F(a, b, 1+m ; x)+H(x),
\end{aligned}
$$


where $H(x)=x^{-m} \sum_{k=0}^{\infty} h_{k} x^{k}$ and $h_{m}=0$, unless either $a$ or $b$ equal $1+m^{\prime}$ with $m^{\prime}$ a natural number $m^{\prime}<m$. In this case the second solution is only a polynomial in $x^{-1}$ and no logarithmic terms are present. In case of $c<1$ instead

$$
\begin{aligned}
& \mathcal{F}_{1}=x^{m} F(a+m, b+m, 1+m ; x), \\
& \mathcal{F}_{2}=\ln x x^{m} F(a+m, b+m, 1+m ; x)+H(x),
\end{aligned}
$$

where $H(x)$ is again some regular expansion without logarithms, unless either $a$ or $b$ equal $-m^{\prime}$ with an integer $m^{\prime}$ such that $0 \leq m^{\prime}<m$, in which case both solutions do not have logarithmic terms also.

A similar phenomenon appears in the Operator Product Expansions (OPE) of vertex operators. Equations of the type (91) appear the conformal blocks of the theory:

$$
<V_{1}\left(z_{1}\right) V_{2}\left(z_{2}\right) V_{2}\left(z_{3}\right) V_{1}\left(z_{4}\right)>=\frac{1}{\left(z_{1}-z_{4}\right)^{2 h}\left(z_{2}-z_{3}\right)^{2 h}} \mathcal{F}(x),
$$

where $x=\left(z_{1}-z_{2}\right)\left(z_{3}-z_{4}\right) /\left(z_{1}-z_{4}\right)\left(z_{3}-z_{2}\right)$ and only the dependence on holomorphic coordinates $z_{i}$ has been written for simplicity, and the $V$ 's are primary fields with the same conformal dimension $h$.

In this case the logarithmic terms of (93) or (94) yield an anomalous OPE:

$$
V_{1}\left(z_{1}\right) V_{2}\left(z_{2}\right)=\ldots+\left(z_{1}-z_{2}\right)^{h_{C}-2 h}\left(\bar{z}_{1}-\bar{z}_{2}\right)^{\bar{h}_{C}-2 \bar{h}}\left[D+C \ln \left|z_{1}-z_{2}\right|^{2}\right]+\ldots
$$

where the dimension $h_{C}$ of the operators $C$ and $D$ is determined by the leading logarithmic terms in the conformal block (95). We have written here both $z$ - and $\bar{z}$-dependences explicitly and it is important to note that the logarithmic term depends on $|z|$, even for chiral fields, because in the full conformal blocks actually $\ln |z|$ appears, as was shown in 13.

The OPE of the stress-energy tensor $T$ with these fields is:

$$
\begin{aligned}
T(z) C(0) & =\frac{h_{C}}{z^{2}} C(0)+\frac{1}{z} \partial_{z} C(0)+\ldots \\
T(z) D(0) & =\frac{h_{C}}{z^{2}} D(0)+\frac{1}{z^{2}} C(0)+\frac{1}{z} \partial_{z} D(0)+\ldots
\end{aligned}
$$

for general logarithmic pair $C$ and $D$ with anomalous dimensions $h$. We see immediately that we have a mixing between $C$ and $D$. The Virasoro operator $L_{0}$ is not diagonal and mixes $C$ and $D$ inside a $2 \times 2$ Jordan cell

$$
L_{0}\left|C>=h_{C}\right| C>; \quad L_{0}\left|D>=h_{C}\right| D>+\mid C>
$$


Substituting (96) into the four-point correlation function yields [13]) the following two-point correlation functions for the fields $C$ and $D$ :

$$
\begin{aligned}
\langle C(x) D(y)\rangle & =\langle C(y) D(x)\rangle=\frac{\kappa}{2(x-y)^{2 h_{C}}} \\
\langle D(x) D(y)\rangle & =\frac{1}{(x-y)^{2 h_{C}}}\left(-\kappa \ln |x-y|^{2}+d\right) \\
\langle C(x) C(y)\rangle & =0
\end{aligned}
$$

This is our defining property of the logarithmic pair.

Note that the constant $d$ is arbitrary and can be changed by shifting $D \rightarrow D+$ const $C$. The coefficient $\kappa$ is defined by the leading logarithmic term in the conformal block.

The $C-D$ mixing has consequences for the string propagator on the world-sheet cylinder between states $\mid m>$ and $|n\rangle$, which is defined as

$$
\int d q d \bar{q}<n\left|q^{L_{0}-1} \bar{q}^{\bar{L}_{0}-1}\right| m>
$$

where $q=\exp (2 \pi i \tau)$ and $\tau$ is the modular parameter. In the usual case, when $L_{0}, \bar{L}_{0}$ are diagonal, one gets after integrating over $\tau$

$$
<n\left|\frac{1}{L_{0}+\bar{L}_{0}-2} \delta\left(L_{0}-\bar{L}_{0}\right)\right| m>,
$$

where $\delta\left(L_{0}-\bar{L}_{0}\right)$ enforce the condition $h=\bar{h}$ for all propagating states. However in the case of logarithmic operators one must take into account the Jordan cell structure of $L_{0}, \bar{L}_{0}$, which in the sector of $\mid C D>$ and $\mid \bar{C} D>$ states leads to

$$
q^{L_{0}}=q^{h_{C}}\left(\begin{array}{cc}
1 & \ln q \\
0 & 1
\end{array}\right) ; \quad q^{\bar{L}_{0}}=\bar{q}^{\bar{h}_{C}}\left(\begin{array}{cc}
1 & \ln \bar{q} \\
0 & 1
\end{array}\right)
$$

We thus have logarithmic factors appearing. Note that $\ln q$ factors arise also in the characters $\operatorname{Tr}_{h} q^{L_{0}}$ as was discussed by Flohr in [37.

For us the propagator takes the form:

$$
\int d q d \bar{q} q^{h_{C}-1} \bar{q}^{\bar{h}_{C}-1}<C D\left|\left(\begin{array}{cc}
1 & \ln q \\
0 & 1
\end{array}\right)\right| C D><\overline{C D}\left|\left(\begin{array}{cc}
1 & \ln \bar{q} \\
0 & 1
\end{array}\right)\right| \overline{C D}>
$$

One has either $\ln q$ or $\ln \bar{q}$ terms for transitions when either $D$ goes to $C$ or $\bar{D}$ goes to $\bar{C}$ and $\ln q \ln \bar{q}=4 \pi^{2}|\tau|^{2}$ for transition $D \bar{D}$ to $C \bar{C}$. 
In our case we are interested in modes with conformal dimension $h_{C} \rightarrow 0$, in which case one obtains extra logarithmic infinities as $q \rightarrow 0$.

\section{Acknowledegments}

We wish to thank Giovanni Amelino-Camelia for many illuminating conversations, and collaboration at an early stage of this work. We would also like to thank John Ellis, Ian Kogan, and John Wheater for numerous discussions and comments. F.L. wishes to thank the Department of (Theoretical) Physics of Oxford University for hospitality.

\section{References}

[1] E. Witten, Nucl. Phys. B460 (1996), 541.

[2] M.B. Green, Nucl. Phys. B293 (1987), 593.

[3] A.B. Zamolodchikov, JETP Lett. 43 (1986), 730; Sov. J. Nucl. Phys. 46 (1987), 1090.

[4] J. Ellis, N.E. Mavromatos and D.V. Nanopoulos, Mod. Phys. Lett. A10 (1995), 1685.

[5] J. Ellis, N.E. Mavromatos and D.V. Nanopoulos, preprint ACT-04/96, CERNTH/96-81, CTP-TAMU-11/96, OUTP-96-15P; hep-th/9605046, Int. J. Mod. Phys. $\mathrm{A}$, in press.

[6] J. Dai, R.G. Leigh and J. Polchinski, Mod. Phys. Lett. A4 (1989), 2073;

J. Polchinski, Phys. Rev. D50 (1994), 6041 ; Phys. Rev. Lett., 75 (1995) 184;

C. Bachas, Phys. Lett. B374 (1996), 37;

J. Polchinski, S. Chaudhuri and C. Johnson, NSF-ITP-96-003 preprint, hepth/9602052, and references therein.

[7] C. Callan and I. Klebanov, Nucl. Phys. B465 (1996), 473.

[8] W. Fischler, S. Paban and M. Rozali, Phys. Lett. B352 (1995), 298; Phys. Lett. B381 (1996), 62;

D. Berenstein, R. Corrado, W. Fischler, S. Paban and M. Rozali, Phys. Lett. B384 (1996), 93.

[9] I.I. Kogan and N.E. Mavromatos, Phys. Lett. B375 (1996), 111.

[10] V. Periwal and O. Tafjord, Phys. Rev. D54 (1996), 3690. 
[11] I. Kogan, N. E. Mavromatos and J.F. Wheater, Oxford preprint OUTP-96-32P, hepth/9606102, Phys. Lett. B in press.

[12] V. Gurarie, Nucl. Phys. B410 (1993), 535.

[13] J.S. Caux, I.I. Kogan and A. Tsvelik, Nucl. Phys. B466 (1996), 444.

[14] I. Antoniades, C. Bachas, J. Ellis, and D.V. Nanopoulos, Phys. Lett. B211 (1988), 393; Nucl. Phys. B328 (1989), 117.

[15] J. Ellis, N.E. Mavromatos and D.V. Nanopoulos, lectures presented at the Erice Summer School 1993; Published in Erice Subnuclear Series (World Sci.) Vol. 31 (1993), 1, hep-th/9403133.

In a different context, see: N.E. Mavromatos and D.V. Nanopoulos, quantph/9512021, Int. J. Mod. Phys. B11 (1996), in press.

[16] I. Kogan, preprint UBCTP-91-13 (1991); Proc. Particles and Fields 91, p. 837, Vancouver 18-21 April 1991 (eds. D. Axen, D. Bryman and M. Comyn, World Sci. 1992); see also Phys. Lett. B265 (1991), 269.

[17] J. Ellis, N.E. Mavromatos and D.V. Nanopoulos, preprint CERN-TH/96-264, OUTP57-P, hep-th/9609238.

[18] S. Hojman and C. Shepley, J. Math. Phys. 32 (1991), 142;

F. Pardo, J. Math. Phys. 30 (1989), 2054.

[19] C. Schmidhuber, Nucl. Phys. 435 (1995), 156.

[20] G. Veneziano, Europhys. Lett. 2 (1986), 199;

D.J. Gross and P.F. Mende, Nucl. Phys. B303 (1988), 407;

D. Amati, M. Ciafaloni and G. Veneziano, Phys. Lett. B216 (1989), 41;

K. Konishi, G. Paffuti and P. Provero, Phys. Lett. B234 (1990), 276.

[21] F. David, Mod. Phys. Lett. A3 (1988), 1651;

J. Distler and H. Kawai, Nucl. Phys. B321 (1989), 509.

[22] I. Klebanov, I. Kogan and A.M. Polyakov, Phys. Rev. Lett. 71 (1993), 3243.

[23] S.H. Shenker, preprint RU-95-53, hep-th/9509132.

[24] J. Polchinski, Nucl. Phys. B307 (1988), 61; ibid B357 (1995), 241.

[25] S. Coleman, Nucl. Phys. B310 (1988), 643.

[26] W. Fischler and L. Susskind, Phys. Lett. B171 (1986), 383; ibid. 173 (1986), 262. 
[27] N.E. Mavromatos and J.L. Miramontes, Phys. Lett. B226 (1989), 291.

[28] N. Gisin, Helv. Phys. Acta 62 (1989), 363;

N. Gisin and I. Percival, J. Phys. A26 (1993), 2233.

[29] G. Amelino-Camelia, J. Ellis, N.E. Mavromatos and D.V. Nanopoulos, hepth/9605211, Int. J. Mod. Phys. A in press, and references therein.

[30] E. Witten, Nucl. Phys. B460 (1996), 335.

[31] B. Zumino Mod. Phys. Lett. A6 (1991), 1225.

[32] S. Doplicher, K. Fredenhagen and J.E. Roberts, Phys. Lett. B331 (1994), 39.

[33] A. Kempf, G. Mangano and R.B. Mann, Phys. Rev. D52 (1995), 1108.

[34] A.V. Yung, Int. J .Mod. Phys. A10 (1995), 1553; ibid. A11 (1996), 951.

[35] E. Witten, Phys. Rev. D44 (1991), 344.

[36] M. Li, Phys. Rev. D54 (1996), 1644.

[37] M.A. Flohr, Int. J. Mod. Phys. A11 (1996), 4147.

[38] E.T.Whittaker and G.N. Watson, A course of Modern Analysis, Cambridge University Press (1927).

[39] I.S. Gradshteyn and I.M. Ryzhik, Tables of Integrals, Series and Products, Academic Press, San Diego, p. 1046, (1992). 